than controls, and plasma lactate levels of glucose intolerant FA patients were higher than controls. By intravenous glucose load, glucose responses did not differ significantly between patients and controls. The results showed that FA is associated with insulin resistance, B-cell deficiency, and type I diabetes. The alterations might be genetically linked or metabolically related to the primary defect in FA. (Finocchiaro G et al. Glucose metabolism alterations in Friedreich's ataxia. Neurology August 1988; 38: 1292-96).

COMMENT. Friedreich's heredodegenerative ataxia, incidence of diabetes in intolerance previously, previously

ataxia, the is recessively inherited.

common during oral glucose tests have been reported but the abnormalities of glucose metabolism have not been studied in detail. The abnormalities of glycemic control in FA may be due to the interplay of insulin resistance and $\mathrm{B}-\mathrm{cell}$ dysfunction or their independent effects.

\title{
INFANTILE GANGLIOSIDOSIS
}

Three sisters with infantile-onset 3 GM1 gangliosidosis are reported from the University of Siens, Italy, and the University of Louvain, Brussels, Belgium. The diagnosis was based on the clinical findings of progressive intellectual deterioration by age 6-8 years, ataxia, spastic tetraparesis, and athetoid-choreiform movements; lysosomal vacuoles in CSF, bone marrow, and conjunctiva; and on decreased activity of serum, leukocyte, and fibroblast B-D-galactosidase and abnormal urinary excretion of oligosaccharides. (Guazzi GC et al. Type 3 (chronic) GM1 gangliosidosis presenting as infanto-choreo-athetotic dementia, without epilepsy, in three sisters. Neurology July 1988; $38: 1124-27$ ).

COMmeNT. Gangliosidosis occurs in 3 forms: 1) infantile, characterized by Hurler's facial features, bony abnormalities, hepatoslenomegaly, cherry-red spot, and progressive neurological signs; 2) late infantile-juvenile, without skeletal changes or marked visceromegaly, but severe intellectual deterioration, ataxia, myoclonic seizures, and retinal degeneration; and 3) dystonic juvenile form. The clinical findings in the present report resembled those in the dystonic form except that the intellectual deterioration was more severe.

\section{TOXIC DISORDERS}

\section{FETAL ALCOHOL SYNDROME}

Hearing and speech and language development in 14 children with fetal alcohol syndrome were evaluated at the Fetal Alcohol Research Center, Wayne State University School of Medicine, Detroit, Michigan, and the Audiology Division, School of Medicine, University of Colorado Health Sciences Center, Denver, Colorado. Recurrent serous otitis media with hearing loss and speech and language problems occurred in 13, and 4 also had sensorineural hearing loss. The IQ was 70-85 in 7 children and below 69 in 7 . The authors conclude that 
hearing disorders are a significant complication of fetal alcohol exposure, probably contributing to the speech and language and learning disabilities commonly associated with the syndrome. (Church MW, Gerkin KP. Hearing disorders in children with fetal alcohol syndrome: findings from case reports. Pediatrics August 1988; 82:147 $-154)$.

COMMENT. As a cause of mental retardation, fetal alcohol syndrome is as prevalent as Down's syndrome and occurs in at least one in 1000 births. Postmortem examination of the brain reveals extensive developmental defects, mostly the consequence of migration anomalies. Heterotopias, lissencephaly, and agenesis of the corpus callosum have been described. Migration anomalies may involve the brain stem as well as the cerebrum, thus accounting for the sensorineural deafness noted in the above study.

\section{VALPROATE TOXICITY}

Gastritis and erosion of the gastric mucosa is reported in 10 children who presented with feeding difficulties after long-term treatment with divalproex sodium (Depakote) and valproic acid (Depakene) at the Departments of Neurology, Pediatrics, and Pediatric Surgery, University of Oklahoma Health Sciences Center, Oklahoma City. Anorexia and refusal to eat were complicated by vomiting in 8 patients, abdominal pain in 5, weight loss in 3, and diarrhea in 2 . Endoscopy confirmed erosive gastritis. Treatment with oral antacids (Malox) and cimetidine resulted in clinical improvement. (Marks WA et al. Gastritis with valproate therapy. Arch Neurol August 1988; $\underline{45}: 903-905$ ).

COMMENT. Gastrointestinal disturbances are common at the initiation of valproate therapy but have not previously been reported following its long term use. The diagnosis of gastritis in mentally retarded patients with epilepsy may be difficult, and delay may lead to gastric erosion, resulting in malnutrition, weight loss, and dehydration. If liver and pancreas disease has been excluded as a side-effect, the treatment of valproate-induced gastritis includes antacids and an alteration of anticonvulsant regimen in some cases.

\section{NEONATAL SEIZURES}

\section{INFANTILE SPASMS, HYPSARRHYTHMIA, AND ADRENOLEUKODYSTROPHY (ALD)}

An 8 1/2 month-old girl with seizures beginning at 5 days, hypsarrhythmia in the EEG, severe retardation, and a clinical diagnosis of infantile spasms was discovered to have biochemical and pathological features of adrenoleukodystrophy, as reported from the John F. Kennedy Institute, Johns Hopkins University, Baltimore, Maryland. Laboratory studies showed elevated plasma levels of very long chain fatty acids, and postmortem examination at 14 months revealed cerebral destructive lesions and adrenal cortex atrophy. Seizure frequency had diminished initially with prednisone 